\title{
Permanence of Diffusive Models for Three Competing Species in Heterogeneous Environments
}

\author{
Benlong $\mathrm{Xu}$ and Zhenzhang $\mathrm{Ni}$ \\ Department of Mathematics, Shanghai Normal University, Shanghai 200234, China \\ Correspondence should be addressed to Benlong Xu; bxu@shnu.edu.cn
}

Received 29 May 2014; Revised 20 July 2014; Accepted 21 July 2014; Published 12 August 2014

Academic Editor: Yongli Song

Copyright (C) 2014 B. Xu and Z. Ni. This is an open access article distributed under the Creative Commons Attribution License, which permits unrestricted use, distribution, and reproduction in any medium, provided the original work is properly cited.

\begin{abstract}
We address the question of the long-term coexistence of three competing species whose dynamics are governed by the partial differential equations. We obtain criteria for permanent coexistence in a Lotka-Volterra system modeling the interaction of three competing species in a bounded habitat whose exterior is lethal to each species. It is also proved that if the intercompeting strength is very weak, the system is always permanent, provided that each single one of the three species can survive in the absence of the two other species.
\end{abstract}

\section{Introduction}

Reaction-diffusion systems are some of the most widely used models for population dynamics in situations where spatial dispersal plays a significant role. Generally speaking, the dynamical behaviors of the systems with two competing species are relatively simple and have been widely investigated in the past years. However, the investigation and knowledge about the systems with three competing species are very limited; see [1-5] for some related results. It is well known, in general, that the dynamical behavior of systems with three competing species may be extremely complex, even in nondiffusive case, namely, ODE case; see, for example, [6-9] and the references therein.

The object of this paper is to study the problem of coexistence for three competing species dispersing through a spatially heterogeneous region. We model the population dynamics of the species with a system of three diffusive LotkaVolterra equations.

The model we consider has the general form

$$
\begin{aligned}
\frac{\partial u_{1}}{\partial t}=\mu_{1} \Delta u_{1}+u_{1} & \left(a_{1}(x)-b_{11}(x) u_{1}\right. \\
& \left.-b_{12}(x) u_{2}-b_{13}(x) u_{3}\right) \quad \text { in } \Omega,
\end{aligned}
$$

$$
\begin{gathered}
\frac{\partial u_{2}}{\partial t}=\mu_{2} \Delta u_{2}+u_{2}\left(a_{2}(x)-b_{21}(x) u_{1}\right. \\
\left.-b_{22}(x) u_{2}-b_{23}(x) u_{3}\right) \quad \text { in } \Omega, \\
\frac{\partial u_{3}}{\partial t}=\mu_{3} \Delta u_{3}+u_{3}\left(a_{3}(x)-b_{31}(x) u_{1}\right. \\
\left.-b_{32}(x) u_{2}-b_{33}(x) u_{3}\right) \text { in } \Omega
\end{gathered}
$$

with $u_{i}=0$ on $\partial u_{i} \times \mathbb{R}_{+}$, where $\Omega \subseteq \mathbb{R}^{N}$ is a bounded domain and $a_{i}(x)>0, b_{i i}(x)>0, b_{i j}(x) \geq 0(i, j=1,2,3, i \neq j)$ are all continuous in $\bar{\Omega}$. The variables $u_{i}$ represent population densities of the competing species. The boundary condition $u_{i}=0$ describes a situation that the boundary of $\Omega$ is lethal to the species.

We will use the criterion of permanence to characterize coexistence. A system is said to be permanent if any solutions with all components positive initially must ultimately enter and remain within a fixed set of positive states that are strictly bounded away from zero in each component. For some investigations of coexistence characterized by permanence, mostly in the case for two interacting species, see [10-12], and also some parts of the books $[13,14]$.

This paper is organized as follows. In Section 2, we introduce some necessary material about semiflow and state 
V. Hutson's average Lyapunov theorem which will be the basic tool in the proof of our main theorem. We also discuss the dissipative property of our systems, which is necessary for using V. Hutson's average Lyapunov function theorem. In Section 3, we establish our main permanent result, Theorem 11. We also prove that if the intercompeting strength is "very weak," the system (1) is always permanent, provided that either single one of the three species can survive with the absence of the two other species. In Section 4, we give some summery and discussion of the results of this paper.

\section{Semiflows and Dissipativity}

In this section, we will make some preparations for establishing our main results in the next section. Firstly, we will introduce some terminologies and results about semiflows (semigroups) for readers' convenience. The materials can be found in $[15]$ or $[13,14]$.

Let $(Y, d)$ be a metric space, with points in $Y$ being denoted by $u, v, \ldots$ and subsets $Y$ by $U, V, \ldots$ The following two unsymmetric distances of sets will be used:

$$
\begin{aligned}
& \bar{d}(U, V)=\sup _{u \in U} d(u, V), \\
& \underline{d}(U, V)=\inf _{u \in U} d(u, V) .
\end{aligned}
$$

The triple $\left(Y, \pi, \mathbb{R}_{+}\right)$is said to be a semiflow (or semigroup), if $\pi: Y \times \mathbb{R}_{+} \rightarrow Y$ is continuous and satisfies:

(i) $\pi(u, 0)=u$,

(ii) $\pi(\pi(u, t), s)=\pi(u, t+s)\left(s, t \in R_{+}\right)$,

for all $u \in Y$. For convenience, we often write $\pi(u, t)=u \cdot t$. The symbols $\gamma^{+}(u)$ and $\omega(u)$ denote the semiorbit through $u$ and the omega limit set of $u$, respectively, and the equivalent expressions for sets are defined by taking unions.

A solution $\phi$ through $u$ is a continuous map $\phi: R \rightarrow Y$ such that $\phi(0)=u$ and $\pi(\phi(\tau), t)=\phi(t+\tau)$ for $t \in \mathbb{R}_{+}, \tau \in$ $\mathbb{R}$. The range of $\phi$ is denoted by $\gamma(u)$ and is called an orbit through $u$.

A set $U$ is said to be forward invariant if $\gamma^{+}(u) \subset U$ and invariant if $\gamma(u) \subset U$. The semiflows is said to be dissipative if there is a bounded set $U$ such that $\lim _{t \rightarrow \infty} d(u \cdot t, U)=0$ for all $u \in Y$. $U$ is said to be a global attractor of the semiflow if it is compact invariant and for all bounded $V, \lim _{t \rightarrow \infty} \bar{d}(V$. $t, U)=0$, where $V \cdot t=\{v \cdot t, v \in V\}$.

Theorem 1. Let $Y$ be complete and suppose that the semiflow is dissipative. Assume that there is a $t_{0} \geq 0$ such that $\pi(\cdot, t)$ is compact for $t>t_{0}$; then there is a nonempty global attractor.

Consider next the concept of permanence in the abstract semiflow context. We suppose that $Y=Y_{0} \cup \partial Y_{0}$, where $Y_{0}$ is open, and assume that $Y_{0}, \partial Y_{0}$ are forward invariant. In relation to the remarks in the introduction, $\partial Y_{0}$ will consist of the states with at least one species identically zero.

Definition 2. The semiflow is said to be permanent if there exists a bounded set $U$ with $\underline{d}\left(U, \partial Y_{0}\right)>0$ such that $\lim _{t \rightarrow \infty} d(v \cdot t, U)=0$ for all $v \in \bar{Y}_{0}$.
A set $U \subset Y_{0}$ is said to be strongly bounded if it is bounded and $\underline{d}\left(U, \partial Y_{0}\right)>0 . \mathscr{A}_{0}$ is said to be a global attractor relative to strongly bounded sets if it is a compact invariant subset of $Y_{0}$ and $\lim _{t \rightarrow \infty} \bar{d}\left(U \cdot t, \mathscr{A}_{0}\right)=0$ for all strongly bounded $U$.

Permanence is obviously an asymptotic property. It can thus be studied by examining the semiflow restricted to a forward invariant set derived from an $\varepsilon$-neighbourhood $B(\mathscr{A}, \varepsilon)$ of the global attractor $\mathscr{A}$ of Theorem 1 . Set then $X=\operatorname{cl} \pi(B(\mathscr{A}, \varepsilon),[1, \infty))$, the closure of $\pi(B(\mathscr{A}, \varepsilon),[1, \infty))$, and take $S=X \cap \partial Y_{0}$. The following Hutson's theorem on average Lyapunov functions is the basic tool for establishing our main theorem in Section 3.

Theorem 3 (see [15]). Assume that the conditions of Theorem 1 hold, and let $X, S$ be as defined above. Suppose that $P: X \backslash S \rightarrow$ $\mathbb{R}_{+}$is continuous, strictly positive, and bounded, and for $u \in S$ define

$$
\alpha(t, u)=\liminf _{v \rightarrow u, v \in X \backslash S}\left(\frac{P(v \cdot t)}{P(v)}\right) .
$$

Then the semiflow is permanent if

$$
\begin{gathered}
\sup _{t>0} \alpha(t, u)>1 \quad u \in \omega(S), \\
\sup _{t>0} \alpha(t, u)>0 \quad u \in S .
\end{gathered}
$$

For our application of the last theorem, we will cast the system (1) to the abstract frame of the semiflow. It is well known that if (1) is simply viewed as a parabolic system, it actually generated a semiflow in $C^{1}(\bar{\Omega})$, and solutions which belong to $C^{1}(\bar{\Omega})$ must have Hölder-continuous second derivatives on $\Omega$ (see [13]). Thus, we may use maximum principles to obtain a priori bounds, even though we will ultimately want to view our semiflow as acting on $C^{1}(\bar{\Omega})$. Then by maximum principle (or comparison theorem), any solution $\left(u_{1}, u_{2}, u_{3}\right)$ of (1) must satisfy a uniform bound of the form $0 \leq u_{i} \leq C_{i}$ for some constants $C_{i}, i=1,2,3$, after finite time. So the semiflow generated by (1) is dissipative. We state it as follows.

Theorem 4. The semiflow generated by the system (1) is dissipative.

\section{Permanent Coexistence Results}

In this section, we establish criteria of permanent coexistence of the system (1). The main tool is Hutson's average Lyapunov function theorem stated in Section 2, and the key step is to set up a suitable average Lyapunov function. To construct the average Lyapunov functions, we must have a detailed knowledge of the $\omega$-limit set of the generated semiflow in the boundary of the positive cone.

The maximum principle implies that solutions of (1) with nonnegative nonzero initial data for a given component will have that component strictly positive in $\Omega$ for all $t>0$. In the case of Dirichlet conditions, such solutions will have normal derivatives on $\partial \Omega$ which are bounded above by a negative 
constant. Hence, the only trajectories which remain in the boundary of the positive cone have one or both components identically zero. Thus, to determine the $\omega$-limit set of the semiflow generated by (1) on the boundary, we need only consider the steady state solutions (equilibrium points) of subsystems of (1).

Let $\lambda_{1}>0$ denote the principal eigenvalue for problem

$$
\begin{gathered}
\Delta \phi+\lambda \phi=0 \quad \text { in } \Omega, \\
\phi=0 \quad \text { on } \partial \Omega .
\end{gathered}
$$

In the rest of this paper, we denote

$$
M_{i}=\max _{x \in \bar{\Omega}} \frac{a_{i}(x)}{b_{i i}(x)} .
$$

The point $(0,0,0)$ is always a steady state, which means that there are no species in the domain. With the absence of two species and only the other one species left, system (1) becomes a scalar equation, and we have the following wellknown result (see [14]).

Lemma 5. Suppose that $a(x)>\mu_{i} \lambda_{1}$ for $x \in \bar{\Omega}$. Then the following problem

$$
\begin{gathered}
u_{i t}=\mu_{i} \Delta u_{i}+u_{i}\left(a_{i}(x)-b_{i i}(x) u_{i}\right) \text { in } \Omega \times(0, \infty), \\
u_{i}=0 \text { on } \partial \Omega \times(0, \infty)
\end{gathered}
$$

for $=1,2,3$, has a unique positive steady state solution $\bar{u}_{i}(x)$ which is globally approximately stable.

Remark 6. Lemma 5 tells us that if $a(x)>\mu_{i} \lambda_{1}$ for $x \in \bar{\Omega}, i=$ $1,2,3$, then either single one of the three species can survive in the absence of the other two species.

Now we consider the subsystem of (1) with only one species to be absent. There are three cases.

Case 1. One has $u_{3} \equiv 0$,

$$
\begin{array}{rr}
u_{1 t}=\mu_{1} \Delta u_{1}+u_{1}\left(a_{1}(x)-b_{11}(x) u_{1}-b_{12}(x) u_{2}\right) & \text { in } \Omega \times(0, \infty), \\
u_{2 t}=\mu_{2} \Delta u_{2}+u_{2}\left(a_{2}(x)-b_{21}(x) u_{1}-b_{22}(x) u_{2}\right) \\
\text { in } \Omega \times(0, \infty), \\
\begin{array}{ll}
u_{1}=u_{2}=0 \quad \text { on } \partial \Omega \times(0, \infty) . &
\end{array}
\end{array}
$$

Case 2. One has $u_{2} \equiv 0$,

$$
\begin{aligned}
& u_{1 t}=\mu_{1} \Delta u_{1}+u_{1}\left(a_{1}(x)-b_{11}(x) u_{1}-b_{13}(x) u_{3}\right) \text { in } \Omega \times(0, \infty), \\
& u_{3 t}=\mu_{3} \Delta u_{3}+u_{3}\left(a_{3}(x)-b_{31}(x) u_{1}-b_{33}(x) u_{3}\right) \\
& \text { in } \Omega \times(0, \infty), \\
& \begin{array}{ll}
u_{1}=u_{3}=0 \quad \text { on } \partial \Omega \times(0, \infty) . &
\end{array}
\end{aligned}
$$

Case 3. One has $u_{1} \equiv 0$,

$$
\begin{aligned}
& u_{2 t}=\mu_{2} \Delta u_{2}+u_{2}\left(a_{2}(x)-b_{22}(x) u_{2}-b_{23}(x) u_{3}\right) \text { in } \Omega \times(0, \infty), \\
& u_{3 t}=\mu_{3} \Delta u_{3}+u_{3}\left(a_{3}(x)-b_{32}(x) u_{2}-b_{33}(x) u_{3}\right) \\
& \text { in } \Omega \times(0, \infty), \\
& \begin{array}{ll}
u_{1}=u_{3}=0 \quad \text { on } \partial \Omega \times(0, \infty) . &
\end{array}
\end{aligned}
$$

With some modification of lower-supper solution methods of parabolic systems (see [14]), it is easy to prove the following results.

Theorem 7. With $M_{i}, i=1,2,3$, as defined in (6),

(1) if

$$
\begin{array}{r}
a_{1}(x)>\mu_{1} \lambda_{1}+M_{2} b_{12}(x), \\
a_{2}(x)>\mu_{2} \lambda_{1}+M_{1} b_{21}(x), \\
\forall x \in \bar{\Omega},
\end{array}
$$

the system (8) has a steady state solution $\left(\widetilde{u}^{(12)}(x)\right.$, $\left.\tilde{u}^{(21)}(x)\right)$ with $\tilde{u}^{(12)}(x)>0, \tilde{u}^{(21)}(x)>0$ for $x \in \Omega$; similarly,

(2) if

$$
\begin{aligned}
& a_{1}(x)>\mu_{1} \lambda_{1}+M_{3} b_{13}(x), \\
& a_{3}(x)>\mu_{3} \lambda_{1}+M_{1} b_{31}(x), \quad \forall x \in \bar{\Omega},
\end{aligned}
$$

the system (9) has a steady state solution $\left(\widetilde{u}^{(13)}(x)\right.$, $\left.\tilde{u}^{(31)}(x)\right)$ with $\tilde{u}^{(13)}(x)>0, \widetilde{u}^{(31)}(x)>0$ for $x \in \Omega$;

(3) if

$$
\begin{aligned}
& a_{2}(x)>\mu_{2} \lambda_{1}+M_{3} b_{23}(x), \\
& a_{3}(x)>\mu_{3} \lambda_{1}+M_{2} b_{32}(x), \quad \forall x \in \bar{\Omega},
\end{aligned}
$$

the system (10) has a steady state solution $\left(\widetilde{u}^{(23)}(x)\right.$, $\left.\tilde{u}^{(32)}(x)\right)$ with $\tilde{u}^{(23)}(x)>0, \widetilde{u}^{(32)}(x)>0$ for $x \in \Omega$.

Proof. We only prove the part (1); the proof of part (2) and part (3) are similar. 
Let $w(x)$ be a positive eigenfunction of the principal eigenvalue $\lambda_{1}$ for the eigenvalue problem (5). Choose $r_{1}>0$ sufficiently small,

$$
\begin{aligned}
& \mu_{1} \Delta\left(r_{1} w(x)\right)+r_{1} w(x) \\
& \times\left[a_{1}(x)-b_{11}(x) r_{1} w(x)-b_{12}(x) u_{2}\right] \\
& \geq r_{1} w(x)\left[a_{1}(x)-\mu_{1} \lambda_{1}\right. \\
&\left.-b_{11}(x) r_{1} w(x)-b_{12}(x) M_{2}\right] \geq 0, \\
& \mu_{1} \Delta\left(M_{1}\right)+M_{1}\left[\left(a_{1}(x)-b_{11}(x) M_{1}-b_{12}(x) u_{2}\right]\right. \\
&=M_{1}\left[\left(a_{1}(x)-b_{11}(x) M_{1}-b_{12}(x) u_{2}\right]\right. \\
& \leq M_{1}\left[\left(a_{1}(x)-b_{11}(x) M_{1}\right] \leq 0\right.
\end{aligned}
$$

for all $0 \leq u_{2} \leq M_{2}$. So, $\left(M_{1}, r_{1} w(x)\right)$ is a set of upper and lower solutions for $u_{1}$ in (8).

Similarly, choose $r_{2}>0$ sufficiently small; $\left(M_{2}, r_{2} w(x)\right)$ is a set of upper and lower solutions for $u_{2}$ in (8).

By coupled upper and lower theorem (see [16, Theorem 1.4-2]), the system (8) has a steady state solution $\left(\widetilde{u}^{(12)}(x), \widetilde{u}^{(21)}(x)\right)$ with $\tilde{u}^{(12)}(x)>0, \widetilde{u}^{(21)}(x)>0$ for $x \in$ $\Omega$.

Remark 8. By the comparison principle, it is easy to see that, in $\Omega$, we have $\tilde{u}^{(12)}, \widetilde{u}^{(13)}<\bar{u}_{1}<M_{1}, \widetilde{u}^{(21)}, \widetilde{u}^{(23)}<\bar{u}_{2}<M_{2}$, and $\tilde{u}^{(31)}, \tilde{u}^{(32)}<\bar{u}_{3}<M_{3}$.

Now, we consider the unique problem of the steady state solutions above.

Let $\theta_{\mu, A(x)}$ denote the unique positive solution of

$$
\begin{gathered}
\mu_{i} \Delta \theta+\theta(A(x)-\theta)=0 \quad \text { in } \Omega, \\
\theta=0 \quad \text { on } \partial \Omega,
\end{gathered}
$$

for any $A(x)>\mu_{i} \lambda_{1}$. For brevity, we denote

$$
\begin{aligned}
& \bar{u}^{(1)}=\theta_{\mu_{1}, a_{1}(x)}, \quad \bar{u}^{(2)}=\theta_{\mu_{2}, a_{2}(x)}, \quad \bar{u}^{(3)}=\theta_{\mu_{3}, a_{3}(x)}, \\
& \underline{u}^{(12)}=\theta_{\mu_{1},\left(a_{1}(x)-M_{2} b_{12}(x)\right)}, \quad \underline{u}^{(21)}=\theta_{\mu_{2},\left(a_{2}(x)-M_{1} b_{21}(x)\right)}, \\
& \underline{u}^{(13)}=\theta_{\mu_{1},\left(a_{1}(x)-M_{3} b_{13}(x)\right)}, \quad \underline{u}^{(31)}=\theta_{\mu_{3},\left(a_{3}(x)-M_{1} b_{31}(x)\right)}, \\
& \underline{u}^{(23)}=\theta_{\mu_{2},\left(a_{2}(x)-M_{3} b_{23}(x)\right)}, \quad \underline{u}^{(32)}=\theta_{\mu_{3},\left(a_{3}(x)-M_{2} b_{32}(x)\right)} .
\end{aligned}
$$

They are all positive functions in $\Omega$.

Theorem 9. Assume that all the hypotheses of Theorem 7 are satisfied.

(1) If

$$
\frac{b_{12}^{2}(x) \bar{u}^{(1)}}{b_{11}^{2}(x) \underline{u}^{(21)}}+2 \frac{b_{12}(x) b_{21}(x)}{b_{11}(x) b_{22}(x)}+\frac{b_{21}^{2}(x) \bar{u}^{(2)}}{b_{22}^{2}(x) \underline{u}^{(12)}}<4,
$$

then the strictly positive steady state solution $\left(\widetilde{u}^{(12)}(x), \widetilde{u}^{(21)}(x)\right)$ of system (8) is unique; similarly
(2) if

$$
\frac{b_{13}^{2}(x) \bar{u}^{(1)}}{b_{11}^{2}(x) \underline{u}^{(31)}}+2 \frac{b_{13}(x) b_{31}(x)}{b_{11}(x) b_{33}(x)}+\frac{b_{31}^{2}(x) \bar{u}^{(3)}}{b_{33}^{2}(x) \underline{u}^{(13)}}<4,
$$

then the steady state solution $\left(\widetilde{u}^{(13)}(x), \widetilde{u}^{(31)}(x)\right)$ of system (9) is unique;

(3) if

$$
\frac{b_{23}^{2}(x) \bar{u}^{(2)}}{b_{22}^{2}(x) \underline{u}^{(32)}}+2 \frac{b_{23}(x) b_{32}(x)}{b_{22}(x) b_{33}(x)}+\frac{b_{32}^{2}(x) \bar{u}^{(3)}}{b_{33}^{2}(x) \underline{u}^{(23)}}<4,
$$

then the steady state solution $\left(\widetilde{u}^{(23)}(x), \widetilde{u}^{(32)}(x)\right)$ of system (10) is unique.

Remark 10. For fixed functions $a_{i}(x)>0, b_{i i}(x)>0$, $(i=1,2,3)$, hypothesis (17) will be satisfied for $b_{12}(x)$, $b_{21}(x) \geq 0$ sufficiently small. This is true because of Hopf's strong maximal value theorem, and also because $\underline{u}^{(12)}$ (resp., $\underline{u}^{(21)}$ ) increases as $b_{12}(x)$ (resp., $\left.b_{21}(x)\right)$ decreases for $x \in$ $\Omega$. Thus $\left(b_{21}^{2}(x) \bar{u}^{(2)} / b_{22}^{2}(x) \underline{u}^{(12)}\right)$ (resp., $b_{12}^{2}(x) \bar{u}^{(1)} / b_{11}^{2}(x) \underline{u}^{(21)}$ ) decreases as $b_{12}(x)$ (resp., $b_{21}(x)$ ) decreases. Similarly, hypotheses (18) and (19) will be satisfied if $b_{13}(x), b_{31}(x)$, $b_{23}(x)$, and $b_{32}(x)$ are sufficiently small.

Proof. We only give the proof of part (1) in Theorem 9, since the arguments of part (2) and part (3) are similar.

Assume that $\left(\widetilde{u}^{(12)}(x), \widetilde{u}^{(21)}(x)\right),\left(\widetilde{v}^{(12)}(x), \widetilde{v}^{(21)}(x)\right)$ are two strictly positive steady state solutions of system (8) in $\Omega$.

Let

$$
\begin{aligned}
& p(x)=\tilde{u}^{(12)}(x)-\widetilde{v}^{(12)}(x), \\
& q(x)=\tilde{u}^{(21)}(x)-\widetilde{v}^{(21)}(x) \\
& I_{12}=b_{11}(x) \widetilde{v}^{(12)}(x) p(x)+b_{12}(x) \widetilde{v}^{(12)}(x) q(x) \\
& I_{21}=b_{21}(x) \tilde{u}^{(21)}(x) p(x)+b_{22}(x) \tilde{u}^{(21)}(x) q(x) ;
\end{aligned}
$$

then

$$
\begin{gathered}
\mu_{1} \Delta p(x)+\left[a_{1}(x)-b_{11}(x) \tilde{u}^{(12)}(x)\right. \\
\left.-b_{12}(x) \tilde{u}^{(21)}(x)\right] p(x)-I_{12}=0 \\
\text { in } \Omega, \\
\mu_{2} \Delta q(x)+\left[a_{2}(x)-b_{21}(x) \widetilde{v}^{(12)}(x)\right. \\
\left.-b_{22}(x) \widetilde{v}^{(21)}(x)\right] q(x)-I_{21}=0
\end{gathered}
$$

in $\Omega$,

$p(x)=q(x)=0 \quad$ on $\partial \Omega$. 
Since $\widetilde{u}^{(12)}(x)$ is a strictly positive solution of

$$
\begin{gathered}
\mu_{1} \Delta \psi+\left[a_{1}(x)-b_{11}(x) \tilde{u}^{(12)}(x)\right. \\
\left.-b_{12}(x) \tilde{u}^{(21)}(x)\right] \psi+\alpha \psi=0 \\
\text { in } \Omega, \\
\psi=0 \quad \text { on } \partial \Omega,
\end{gathered}
$$

with $\alpha=0$, the number $\alpha=0$ must be the smallest eigenvalue of the above problem. Moreover, by variational properties, we have

$$
\begin{aligned}
\int_{\Omega} z\left(-\mu_{1} \Delta z-[\right. & a_{1}(x)-b_{11}(x) \tilde{u}^{(12)}(x) \\
& \left.\left.-b_{12}(x) \tilde{u}^{(21)}(x)\right] z\right) d x \geq 0,
\end{aligned}
$$

for any $z \in C^{2}(\bar{\Omega})$ which vanishes on $\partial \Omega$. Similarly, since $\widetilde{v}^{(21)}(x)$ is strictly positive solution of

$$
\begin{gathered}
\mu_{2} \Delta \psi+\left[a_{2}(x)-b_{21}(x) \widetilde{v}^{(12)}(x)\right. \\
\left.-b_{22}(x) \widetilde{v}^{(21)}(x)\right] \psi+\alpha \psi=0 \\
\text { in } \Omega, \\
\psi=0 \quad \text { on } \partial \Omega,
\end{gathered}
$$

with $\alpha=0$, the number $\alpha=0$ must be the smallest eigenvalue of the above problem. Moreover,

$$
\begin{aligned}
\int_{\Omega} z\left(-\mu_{2} \Delta z-[\right. & a_{2}(x)-b_{21}(x) \widetilde{v}^{(12)}(x) \\
& \left.\left.-b_{22}(x) \widetilde{v}^{(21)}(x)\right] z\right) d x \geq 0,
\end{aligned}
$$

for any $z \in C^{2}(\bar{\Omega})$ which vanishes on $\partial \Omega$. Multiplying the first equation of (21) by $-p(x)$, the second by $-q(x)$, integrating over $\Omega$, and adding, we deduce from (23) and (25) that

$$
\begin{aligned}
& \int_{\Omega}\left[b_{11}(x) \widetilde{v}^{(12)}(x) p^{2}(x)\right. \\
& \quad+\left(b_{12}(x) \widetilde{v}^{(12)}(x)+b_{21}(x) \tilde{u}^{(21)}(x)\right) p(x) q(x) \\
& \left.\quad+b_{22}(x) \tilde{u}^{(21)}(x) q^{2}(x)\right] d x \leq 0 .
\end{aligned}
$$

By comparison of scalar equations using upper and lower solutions we can readily obtain for $i=1,2, x \in \Omega$,

$$
\begin{array}{ll}
\left(\frac{1}{b_{11}(x)}\right) \underline{u}^{(12)} \leq \tilde{u}^{(12)}, & \widetilde{v}^{(12)} \leq\left(\frac{1}{b_{11}(x)}\right) \bar{u}^{(1)}, \\
\left(\frac{1}{b_{22}(x)}\right) \underline{u}^{(21)} \leq \tilde{u}^{(21)}, & \widetilde{v}^{(21)} \leq\left(\frac{1}{b_{22}(x)}\right) \bar{u}^{(2)} .
\end{array}
$$

From (27), we have

$$
\begin{gathered}
\frac{b_{22}(x) b_{12}^{2}(x) \bar{u}^{(1)}}{b_{11}(x) \underline{u}^{(21)}}+2 b_{12}(x) b_{21}(x)+\frac{b_{11}(x) b_{21}^{2}(x) \bar{u}^{(2)}}{b_{22}(x) \underline{u}^{(12)}} \\
>b_{12}^{2}(x) \frac{\widetilde{v}^{(12)}(x)}{\widetilde{u}^{(21)}(x)}+2 b_{12}(x) b_{21}(x)+b_{21}^{2}(x) \frac{\widetilde{u}^{(21)}(x)}{\widetilde{v}^{(12)}(x)}
\end{gathered}
$$

in $\Omega$. It follows from (17) that

$$
\begin{aligned}
& b_{12}^{2}(x) \frac{\widetilde{v}^{(12)}(x)}{\widetilde{u}^{(21)}(x)}+2 b_{12}(x) b_{21}(x) \\
& \quad+b_{21}^{2}(x) \frac{\widetilde{u}^{(21)}(x)}{\widetilde{v}^{(12)}(x)}<4 b_{11}(x) b_{22}(x), \quad \text { in } \Omega .
\end{aligned}
$$

Then it is easy to see that the quadratic expression in the integrand of (26) is positive definite for each $x \in \Omega$. Consequently, we must have $p(x)$ and $q(x)$ identically equal to zero in $\Omega$. That is $\left(\widetilde{u}^{(12)}(x), \widetilde{u}^{(21)}(x)\right) \equiv\left(\widetilde{v}^{(12)}(x), \widetilde{v}^{(21)}(x)\right)$ in $\Omega$.

Now we are ready to develop the permanent coexistence results of the system (1). To construct the average Lyapunov functions, we will use the positive eigenfunctions $\psi_{1}, \psi_{2}$, and $\psi_{3}$ corresponding to the principal eigenvalues $\sigma_{1}, \sigma_{2}$, and $\sigma_{3}$ of

$$
\begin{gathered}
\mu_{1} \Delta \psi_{1}+\psi_{1}\left(a_{1}(x)-b_{12}(x) \tilde{u}^{(23)}-b_{13}(x) \tilde{u}^{(32)}\right) \\
=\sigma \psi_{1} \quad \text { in } \Omega, \\
\psi_{1}=0 \quad \text { on } \partial \Omega, \\
\mu_{2} \Delta \psi_{2}+\psi_{2}\left(a_{2}(x)-b_{21}(x) \tilde{u}^{(13)}-b_{23}(x) \tilde{u}^{(31)}\right) \\
=\sigma \psi_{2} \quad \text { in } \Omega, \\
\psi_{2}=0 \quad \text { on } \partial \Omega, \\
\mu_{3} \Delta \psi_{3}+\psi_{3}\left(a_{3}(x)-b_{31}(x) \tilde{u}^{(12)}-b_{32}(x) \tilde{u}^{(21)}\right) \\
=\sigma \psi_{3} \quad \text { in } \Omega,
\end{gathered}
$$

respectively.

Theorem 11. Suppose that (11)-(13), (17)-(19) hold, and the principal eigenvalues $\sigma_{1}, \sigma_{2}$, and $\sigma_{3}$ of (30), (31), and (32), respectively, are all positive. Assume also that

$$
\begin{aligned}
\sigma_{1} & +b_{12}(x) \tilde{u}^{(23)}+b_{13}(x) \tilde{u}^{(32)} \\
> & \max \left\{b_{12}(x) \bar{u}_{2}, b_{13}(x) \bar{u}_{3}\right\}, \\
\sigma_{2} & +b_{21}(x) \tilde{u}^{(13)}+b_{23}(x) \tilde{u}^{(31)} \\
& >\max \left\{b_{21}(x) \bar{u}_{1}, b_{23}(x) \bar{u}_{3}\right\}, \\
\sigma_{3} & +b_{31}(x) \tilde{u}^{(12)}+b_{32}(x) \tilde{u}^{(21)} \\
& >\max \left\{b_{31}(x) \bar{u}_{1}, b_{32}(x) \bar{u}_{2}\right\},
\end{aligned}
$$

for all $x \in \bar{\Omega}$; then the semiflow on $\left[C_{0}^{1}(\bar{\Omega})\right]^{3}$ generated by (1) under homogeneous Dirichlet boundary conditions is permanent.

Proof. The hypotheses imply that (1) is dissipative and that the $\omega$-limit set in the boundary of the positive cone consists 
of the following seven cases: $(0,0,0),\left(\bar{u}_{1}, 0,0\right),\left(0, \bar{u}_{2}, 0\right)$, $\left(0,0, \bar{u}_{3}\right),\left(\widetilde{u}^{(12)}, \widetilde{u}^{(21)}, 0\right),\left(\widetilde{u}^{(13)}, 0, \widetilde{u}^{(31)}\right)$, and $\left(0, \widetilde{u}^{(23)}, \widetilde{u}^{(32)}\right)$. Thus we need only find a suitable average Lyapunov function to conclude permanence. Since the semiflow is dissipative, we may restrict our attention to a bounded absorbing subset $X$ of the positive cone in $\left[C_{0}^{1}(\bar{\Omega})\right]^{3}$ that contains the global attractor whose existence is asserted in Theorem 1. Let $S$ denote the intersection of $X$ with the boundary of the positive cone. Choose eigenfunctions $\psi_{1}, \psi_{2}, \psi_{3}>0$ for (30), (31), and (32), respectively, and define

$$
\begin{gathered}
P\left(\left(v_{1}, v_{2}, v_{3}\right)\right) \\
=\left(\int_{\Omega} \psi_{1} v_{1} d x\right)\left(\int_{\Omega} \psi_{2} v_{2} d x\right)\left(\int_{\Omega} \psi_{3} v_{3} d x\right) \\
=\exp \left[\log \int_{\Omega} \psi_{1} v_{1} d x+\log \int_{\Omega} \psi_{2} v_{2} d x\right. \\
\left.+\log \int_{\Omega} \psi_{3} v_{3} d x\right] .
\end{gathered}
$$

We have for $\left(u_{1}, u_{2}, u_{3}\right) \in S$ that

$$
\alpha\left(t,\left(u_{1}, u_{2}, u_{3}\right)\right)=\liminf _{\substack{\left(v_{1}, v_{2}, v_{3}\right) \rightarrow\left(u_{1}, u_{2}, u_{3}\right) \\\left(v_{1}, v_{2}, v_{3}\right) \in X \backslash S}} \frac{P\left(\left(v_{1}, v_{2}, v_{3}\right) \cdot t\right)}{P\left(\left(v_{1}, v_{2}, v_{3}\right)\right)}
$$

we need $\sup _{t>0} \alpha\left(t,\left(u_{1}, u_{2}, u_{3}\right)\right)>0$ for $\left(u_{1}, u_{2}, u_{3}\right) \in S$ and $\sup _{t>0} \alpha\left(t,\left(u_{1}, u_{2}, u_{3}\right)\right)>1$ for $\left(u_{1}, u_{2}, u_{3}\right) \in \omega(S)$. Let $\left(w_{1}(t), w_{2}(t), w_{3}(t)\right)=\left(\left(v_{1}, v_{2}, v_{3}\right) \cdot t\right)$. Computation using $(34)$ yields

$$
\begin{gathered}
\frac{P\left(\left(v_{1}, v_{2}, v_{3}\right) \cdot t\right)}{P\left(\left(v_{1}, v_{2}, v_{3}\right)\right)} \\
=\exp \left[\left(\left.\log \int_{\Omega} \psi_{1} w_{1} d x\right|_{t}-\left.\log \int_{\Omega} \psi_{1} w_{1} d x\right|_{0}\right)\right. \\
+\left(\left.\log \int_{\Omega} \psi_{2} w_{2} d x\right|_{t}-\left.\log \int_{\Omega} \psi_{2} w_{2} d x\right|_{0}\right) \\
\left.+\left(\left.\log \int_{\Omega} \psi_{3} w_{3} d x\right|_{t}-\left.\log \int_{\Omega} \psi_{3} w_{3} d x\right|_{0}\right)\right] \\
=\exp \left[\int_{0}^{t}\left(\frac{\int_{\Omega} \psi_{1} w_{1 t} d x}{\int_{\Omega} \psi_{1} w_{1} d x}\right)+\int_{0}^{t}\left(\frac{\int_{\Omega} \psi_{2} w_{2 t} d x}{\int_{\Omega} \psi_{2} w_{2} d x}\right)\right. \\
\left.+\int_{0}^{t}\left(\frac{\int_{\Omega} \psi_{3} w_{3 t} d x}{\int_{\Omega} \psi_{3} w_{3} d x}\right)\right] .
\end{gathered}
$$

We have for $t>0$

$$
\begin{aligned}
& \int_{\Omega} \psi_{1} w_{1 t} d x \\
& =\int_{\Omega} \psi_{1}\left[\mu_{1} \Delta w_{1}+w_{1}\left(a_{1}(x)-b_{11}(x) w_{1}\right.\right. \\
& \left.\left.-b_{12}(x) w_{2}-b_{13}(x) w_{3}\right)\right] d x \\
& =\int_{\Omega}\left[\left(\mu_{1} \Delta \psi_{1}\right) w_{1}+\left(a_{1}(x)-b_{11}(x) w_{1}\right.\right. \\
& \left.\left.-b_{12}(x) w_{2}-b_{13}(x) w_{3}\right) \psi_{1} w_{1}\right] d x \\
& =\int_{\Omega}\left[\sigma_{1}-\left(a_{1}(x)-b_{12}(x) \tilde{u}^{(23)}-b_{13}(x) \tilde{u}^{(32)}\right)\right. \\
& +\left(a_{1}(x)-b_{11}(x) w_{1}-b_{12}(x) w_{2}\right. \\
& \left.\left.-b_{13}(x) w_{3}\right)\right] \psi_{1} w_{1} d x \\
& =\int_{\Omega}\left[\sigma_{1}-b_{11}(x) w_{1}-b_{12}(x)\left(w_{2}-\tilde{u}^{(23)}\right)\right. \\
& \left.-b_{13}(x)\left(w_{3}-\tilde{u}^{(32)}\right)\right] \psi_{1} w_{1} d x .
\end{aligned}
$$

Similarly,

$$
\begin{gathered}
\int_{\Omega} \psi_{2} w_{2 t} d x \\
=\int_{\Omega}\left[\sigma_{2}-\left(a_{2}(x)-b_{21}(x) \tilde{u}^{(13)}-b_{23}(x) \tilde{u}^{(31)}\right)\right. \\
+\left(a_{2}(x)-b_{22}(x) w_{2}-b_{21}(x) w_{1}\right. \\
\left.\left.\quad-b_{23}(x) w_{3}\right)\right] \psi_{2} w_{2} d x \\
=\int_{\Omega}\left[\sigma_{2}-b_{22}(x) w_{2}-b_{21}(x)\left(w_{1}-\tilde{u}^{(13)}\right)\right. \\
\left.-b_{23}(x)\left(w_{3}-\tilde{u}^{(31)}\right)\right] \psi_{2} w_{2} d x, \\
\int_{\Omega} w_{3 t} d x \\
=\int_{\Omega}\left[\sigma_{3}-\left(a_{3}(x)-b_{31}(x) \tilde{u}^{(12)}-b_{32}(x) \tilde{u}^{(21)}\right)\right. \\
+\left(a_{3}(x)-b_{33}(x) w_{3}-b_{31}(x) w_{1}\right. \\
\left.\left.-b_{32}(x) w_{2}\right)\right] \psi_{2} w_{2} d x \\
=\int_{\Omega}\left[\sigma_{3}-b_{33}(x) w_{3}-b_{31}(x)\left(w_{1}-\tilde{u}^{(12)}\right)\right. \\
\left.\left.w_{2}-\tilde{u}^{(21)}\right)\right] \psi_{3} w_{3} d x .
\end{gathered}
$$


Since $X$ is bounded,

$$
\begin{aligned}
\sigma_{1} & -b_{11}(x) w_{1}-b_{12}(x)\left(w_{2}-\tilde{u}^{(23)}\right) \\
& -b_{13}(x)\left(w_{3}-\widetilde{u}^{(32)}\right), \\
\sigma_{2} & -b_{22}(x) w_{2}-b_{21}(x)\left(w_{1}-\widetilde{u}^{(13)}\right) \\
& -b_{23}(x)\left(w_{3}-\widetilde{u}^{(31)}\right), \\
\sigma_{3} & -b_{33}(x) w_{3}-b_{31}(x)\left(w_{1}-\widetilde{u}^{(12)}\right) \\
& -b_{32}(x)\left(w_{2}-\widetilde{u}^{(21)}\right)
\end{aligned}
$$

are bounded below on $X \backslash S$, so that the ratios $\left(\int_{\Omega} \psi_{i} w_{i t} d x / \int_{\Omega} \psi_{i} w_{i} d x\right)$ are bounded below. It follows that $P\left(\left(v_{1}, v_{2}, v_{3}\right) \cdot t\right) / P\left(\left(v_{1}, v_{2}, v_{3}\right)\right)$ has a strictly positive lower bounded since the expression inside the exponential in (36) is bounded away from $-\infty$. To see what happens as $\left(v_{1}, v_{2}, v_{3}\right) \rightarrow \omega(S)$, we must examine how

$$
\begin{aligned}
& \int_{\Omega}\left[\sigma_{1}-b_{11}(x) w_{1}-b_{12}(x)\left(w_{2}-\tilde{u}^{(23)}\right)\right. \\
& \left.\quad-b_{13}(x)\left(w_{3}-\tilde{u}^{(32)}\right)\right] \psi_{1} w_{1} d x \\
& \times\left(\int_{\Omega} \psi_{1} w_{1} d x\right)^{-1} \\
& +\int_{\Omega}\left[\sigma_{2}-b_{22}(x) w_{2}-b_{21}(x)\left(w_{1}-\tilde{u}^{(13)}\right)\right. \\
& \left.\quad-b_{23}(x)\left(w_{3}-\tilde{u}^{(31)}\right)\right] \psi_{2} w_{2} d x \\
& \times\left(\int_{\Omega} \psi_{2} w_{2} d x\right)^{-1} \\
& +\int_{\Omega}\left[\sigma_{3}-b_{33}(x) w_{3}-b_{31}(x)\left(w_{1}-\tilde{u}^{(12)}\right)\right. \\
& \left.\quad-b_{32}(x)\left(w_{2}-\tilde{u}^{(21)}\right)\right] \psi_{3} w_{3} d x \\
& \times\left(\int_{\Omega} \psi_{3} w_{3} d x\right)^{-1}
\end{aligned}
$$

behaves as $\left(v_{1}, v_{2}, v_{3}\right) \rightarrow\left(u_{1}, u_{2}, u_{3}\right) \in \omega(S),\left(v_{1}, v_{2}, v_{3}\right) \in$ $X \backslash S$. By the continuity of the semiflow $\pi, w_{1}, w_{2}$, and $w_{3}$ will be uniformly close to $v_{1}, v_{2}$, and $v_{3}$, respectively, for $t>0$ sufficiently small. Hence, if we can show that the expression

$$
\begin{aligned}
& \sigma^{*}\left(v_{1}, v_{2}, v_{3}\right) \\
& =\int_{\Omega}\left[\sigma_{1}-b_{11}(x) v_{1}-b_{12}(x)\left(v_{2}-\widetilde{u}^{(23)}\right)\right. \\
& \left.\quad-b_{13}(x)\left(v_{3}-\widetilde{u}^{(32)}\right)\right] \psi_{1} v_{1} d x \\
& \quad \times\left(\int_{\Omega} \psi_{1} v_{1} d x\right)^{-1} \\
& +\int_{\Omega}\left[\sigma_{2}-b_{22}(x) v_{2}-b_{21}(x)\left(v_{1}-\widetilde{u}^{(13)}\right)\right. \\
& \left.\quad-b_{23}(x)\left(v_{3}-\widetilde{u}^{(31)}\right)\right] \psi_{2} v_{2} d x
\end{aligned}
$$

$$
\begin{aligned}
& \times\left(\int_{\Omega} \psi_{2} v_{2} d x\right)^{-1} \\
& +\int_{\Omega}\left[\sigma_{3}-b_{33}(x) v_{3}-b_{31}(x)\left(v_{1}-\widetilde{u}^{(12)}\right)\right. \\
& \left.\quad-b_{32}(x)\left(v_{2}-\widetilde{u}^{(21)}\right)\right] \psi_{3} v_{3} d x \\
& \times\left(\int_{\Omega} \psi_{3} v_{3} d x\right)^{-1}
\end{aligned}
$$

always has a positive liminf as $\left(v_{1}, v_{2}, v_{3}\right) \rightarrow\left(u_{1}, u_{2}, u_{3}\right) \in$ $\omega(S)$, then by (35)-(39), we have $\alpha\left(t,\left(u_{1}, u_{2}, u_{3}\right)\right)>1$ for $\left(u_{1}, u_{2}, u_{3}\right) \in \omega(S)$ and $t$ sufficiently small.

If we let $\left(v_{1}, v_{2}, v_{3}\right) \rightarrow(0,0,0)$, then

$$
\begin{gathered}
\sigma_{1}-b_{11}(x) v_{1}-b_{12}(x)\left(v_{2}-\tilde{u}^{(23)}\right)-b_{13}(x)\left(v_{3}-\widetilde{u}^{(32)}\right) \\
\longrightarrow \sigma_{1}+b_{12}(x) \tilde{u}^{(23)}+b_{13}(x) \tilde{u}^{(32)} \geq \sigma_{1}>0, \\
\sigma_{2}-b_{22}(x) v_{2}-b_{21}(x)\left(v_{1}-\tilde{u}^{(13)}\right)-b_{23}(x)\left(v_{3}-\widetilde{u}^{(31)}\right) \\
\longrightarrow \sigma_{2}+b_{21}(x) \tilde{u}^{(13)}+b_{23}(x) \tilde{u}^{(31)} \geq \sigma_{2}>0, \\
\sigma_{3}-b_{33}(x) v_{3}-b_{31}(x)\left(v_{1}-\widetilde{u}^{(12)}\right)-b_{32}(x)\left(v_{2}-\widetilde{u}^{(21)}\right) \\
\longrightarrow \sigma_{3}+b_{31}(x) \tilde{u}^{(12)}+b_{32}(x) \tilde{u}^{(21)} \geq \sigma_{3}>0 .
\end{gathered}
$$

Then

$$
\liminf _{\substack{\left(v_{1}, v_{2}, v_{3}\right) \rightarrow(0,0,0) \\\left(v_{1}, v_{2}, v_{3}\right) \in X \backslash S}} \sigma^{*}\left(v_{1}, v_{2}, v_{3}\right) \geq \sigma_{1}+\sigma_{2}+\sigma_{3}>0 .
$$

As $\left(v_{1}, v_{2}, v_{3}\right) \rightarrow\left(\bar{u}_{1}, 0,0\right)$,

$$
\begin{gathered}
\int_{\Omega}\left[\sigma_{1}-\left(a_{1}(x)-b_{12}(x) \tilde{u}^{(23)}-b_{13}(x) \tilde{u}^{(32)}\right)\right. \\
+\left(a_{1}(x)-b_{11}(x) v_{1}-b_{12}(x) v_{2}\right. \\
\left.\left.\quad-b_{13}(x) v_{3}\right)\right] \psi_{1} v_{1} d x \\
\longrightarrow \int_{\Omega}\left[\sigma_{1}-\left(a_{1}(x)-b_{12}(x) \tilde{u}^{(23)}-b_{13}(x) \tilde{u}^{(32)}\right)\right. \\
\left.+\left(a_{1}(x)-b_{11}(x) \bar{u}_{1}\right)\right] \psi_{1} \bar{u}_{1} d x .
\end{gathered}
$$

Also

$$
\begin{aligned}
\int_{\Omega} & \left(a_{1}(x)-b_{11}(x) \bar{u}_{1}\right) \psi_{1} \bar{u}_{1} d x \\
= & -\int_{\Omega} \psi_{1} \mu_{1} \Delta \bar{u}_{1} d x \\
= & -\int_{\Omega} \bar{u}_{1} \mu_{1} \Delta \psi_{1} d x \\
=-\int_{\Omega} \bar{u}_{1} \psi_{1}\left[\sigma_{1}-\left(a_{1}(x)-b_{12}(x) \tilde{u}^{(23)}\right.\right. & \left.\left.-b_{13}(x) \tilde{u}^{(32)}\right)\right] d x
\end{aligned}
$$


so that as $\left(v_{1}, v_{2}, v_{3}\right) \rightarrow\left(\bar{u}_{1}, 0,0\right)$,

$$
\begin{gathered}
\int_{\Omega}\left[\sigma_{1}-b_{11}(x) v_{1}-b_{12}(x)\left(v_{2}-\tilde{u}^{(23)}\right)\right. \\
\left.\quad-b_{13}(x)\left(v_{3}-\tilde{u}^{(32)}\right)\right] \psi_{1} v_{1} d x \rightarrow 0 .
\end{gathered}
$$

Since $\int_{\Omega} \psi_{1} v_{1} d x \rightarrow \int_{\Omega} \psi_{1} \bar{u}_{1} d x>0$, the first ratio of integrals in $(42)$ has limit 0 as $\left(v_{1}, v_{2}, v_{3}\right) \rightarrow\left(\bar{u}_{1}, 0,0\right)$. For the latter two ratios of integrals, note that as $\left(v_{1}, v_{2}, v_{3}\right) \rightarrow\left(\bar{u}_{1}, 0,0\right)$.

By the assumption (33), we have

$$
\begin{aligned}
& \sigma_{2}-b_{21}(x)\left(\bar{u}_{1}-\tilde{u}^{(13)}\right)+b_{23}(x) \tilde{u}^{(31)}>0, \\
& \sigma_{3}-b_{31}(x)\left(\bar{u}_{1}-\widetilde{u}^{(12)}\right)+b_{32}(x) \tilde{u}^{(21)}>0 .
\end{aligned}
$$

Hence we have

$$
\liminf _{\substack{\left(v_{1}, v_{2}, v_{3}\right) \rightarrow\left(\bar{u}_{1}, 0,0\right) \\\left(v_{1}, v_{2}, v_{3}\right) \in X \backslash S}} \sigma^{*}\left(v_{1}, v_{2}, v_{3}\right)>0 .
$$

As $\left(v_{1}, v_{2}, v_{3}\right) \rightarrow\left(0, \bar{u}_{2}, 0\right)$, computations similar to (45) and (46) show that

$$
\begin{gathered}
\int_{\Omega}\left[\sigma_{2}-b_{22}(x) v_{2}-b_{21}(x)\left(v_{1}-\tilde{u}^{(13)}\right)\right. \\
\left.-b_{23}(x)\left(v_{3}-\widetilde{u}^{(31)}\right)\right] \psi_{2} v_{2} d x \\
\times\left(\int_{\Omega} \psi_{3} v_{3} d x\right)^{-1} \longrightarrow 0
\end{gathered}
$$

By assumption (33), we have

$$
\begin{aligned}
& \sigma_{1}-b_{12}(x)\left(\bar{u}_{2}-\tilde{u}^{(23)}\right)+b_{13}(x) \tilde{u}^{(32)}>0, \\
& \sigma_{3}+b_{31}(x) \tilde{u}^{(12)}-b_{32}(x)\left(\bar{u}_{2}-\widetilde{u}^{(21)}\right)>0 .
\end{aligned}
$$

Hence we have

$$
\liminf _{\substack{\left(v_{1}, v_{2}, v_{3}\right) \rightarrow\left(0, \bar{u}_{2}, 0\right) \\\left(v_{1}, v_{2}, v_{3}\right) \in X \backslash S}} \sigma^{*}\left(v_{1}, v_{2}, v_{3}\right)>0 .
$$

As $\left(v_{1}, v_{2}, v_{3}\right) \rightarrow\left(0,0, \bar{u}_{3}\right)$, computations similar to (45) and (46) show that

$$
\begin{gathered}
\int_{\Omega}\left[\sigma_{3}-b_{33}(x) v_{3}-b_{31}(x)\left(v_{1}-\tilde{u}^{(12)}\right)\right. \\
\left.-b_{32}(x)\left(v_{2}-\tilde{u}^{(21)}\right)\right] \psi_{3} v_{3} d x \\
\times\left(\int_{\Omega} \psi_{3} v_{3} d x\right)^{-1} \longrightarrow 0 .
\end{gathered}
$$

By assumption (33), we get

$$
\begin{aligned}
& \sigma_{1}+b_{12}(x) \tilde{u}^{(23)}-b_{13}(x)\left(\bar{u}_{3}-\widetilde{u}^{(32)}\right)>0, \\
& \sigma_{2}+b_{21}(x) \tilde{u}^{(13)}-b_{23}(x)\left(\bar{u}_{3}-\widetilde{u}^{(31)}\right)>0 .
\end{aligned}
$$

Hence we have

$$
\liminf _{\substack{\left(v_{1}, v_{2}, v_{3}\right) \rightarrow\left(0,0, \bar{u}_{3}\right) \\\left(v_{1}, v_{2}, v_{3}\right) \in X \backslash S}} \sigma^{*}\left(v_{1}, v_{2}, v_{3}\right)>0 .
$$

$$
\begin{aligned}
& \text { As }\left(v_{1}, v_{2}, v_{3}\right) \rightarrow\left(\tilde{u}^{(12)}, \tilde{u}^{(21)}, 0\right), \\
& \begin{aligned}
\int_{\Omega}\left[\sigma_{1}-\left(a_{1}(x)-b_{12}(x) \tilde{u}^{(23)}-b_{13}(x) \tilde{u}^{(32)}\right)\right. \\
\quad+\left(a_{1}(x)-b_{11}(x) v_{1}-b_{12}(x) v_{2}\right. \\
\left.\left.\quad-b_{13}(x) v_{3}\right)\right] \psi_{1} v_{1} d x \\
\longrightarrow \int_{\Omega}\left[\sigma_{1}-\left(a_{1}(x)-b_{12}(x) \tilde{u}^{(23)}-b_{13}(x) \tilde{u}^{(32)}\right)\right. \\
\left.\quad+\left(a_{1}(x)-b_{11}(x) \tilde{u}^{(12)}-b_{12}(x) \tilde{u}^{(21)}\right)\right] \psi_{1} \tilde{u}^{(12)} d x .
\end{aligned}
\end{aligned}
$$

Also

$$
\begin{aligned}
& \int_{\Omega}\left[a_{1}(x)-b_{11}(x) \tilde{u}^{(12)}-b_{12}(x) \tilde{u}^{(21)}\right] \psi_{1} \tilde{u}^{(12)} d x \\
& =-\int_{\Omega} \psi_{1} \mu_{1} \Delta \tilde{u}^{(12)} d x \\
& =-\int_{\Omega} \tilde{u}^{(12)} \mu_{1} \Delta \psi_{1} d x \\
& =-\int_{\Omega} \tilde{u}^{(12)} \psi_{1}\left[\sigma_{1}-\left(a_{1}(x)-b_{12}(x) \tilde{u}^{(23)}\right.\right. \\
& \left.\left.\quad-b_{13}(x) \tilde{u}^{(32)}\right)\right] d x
\end{aligned}
$$

so that as $\left(v_{1}, v_{2}, v_{3}\right) \rightarrow\left(\widetilde{u}^{(12)}, \widetilde{u}^{(21)}, 0\right)$,

$$
\begin{gathered}
\int_{\Omega}\left[\sigma_{1}-b_{11}(x) v_{1}-b_{12}(x)\left(v_{2}-\tilde{u}^{(23)}\right)\right. \\
\left.-b_{13}(x)\left(v_{3}-\tilde{u}^{(32)}\right)\right] \psi_{1} v_{1} d x \\
\quad \times\left(\int_{\Omega} \psi_{1} v_{1} d x\right)^{-1} \longrightarrow 0 .
\end{gathered}
$$

For the second ratio of integrals,

$$
\begin{aligned}
& \int_{\Omega}\left[\sigma_{2}-\left(a_{2}(x)-b_{21}(x) \tilde{u}^{(13)}-b_{23}(x) \tilde{u}^{(31)}\right)\right. \\
& \left.+\left(a_{2}(x)-b_{21}(x) v_{1}-b_{22}(x) v_{2}-b_{23}(x) v_{3}\right)\right] \psi_{2} v_{2} d x \\
& \longrightarrow \int_{\Omega}\left[\sigma_{2}-\left(a_{2}(x)-b_{21}(x) \tilde{u}^{(13)}-b_{23}(x) \tilde{u}^{(31)}\right)\right. \\
& \left.\quad+\left(a_{2}(x)-b_{21}(x) \tilde{u}^{(12)}-b_{22}(x) \tilde{u}^{(21)}\right)\right] \psi_{2} \tilde{u}^{(21)} d x .
\end{aligned}
$$


Also

$$
\begin{aligned}
& \int_{\Omega}\left[a_{2}(x)-b_{21}(x) \tilde{u}^{(12)}-b_{22}(x) \tilde{u}^{(21)}\right] \psi_{2} \tilde{u}^{(12)} d x \\
& =-\int_{\Omega} \psi_{2} \mu_{2} \Delta \tilde{u}^{(21)} d x \\
& =-\int_{\Omega} \tilde{u}^{(21)} \mu_{2} \Delta \psi_{2} d x \\
& =-\int_{\Omega} \tilde{u}^{(21)} \psi_{2}\left[\sigma_{2}-\left(a_{2}(x)-b_{21}(x) \tilde{u}^{(13)}\right.\right. \\
& \left.\left.\quad-b_{23}(x) \tilde{u}^{(31)}\right)\right] d x
\end{aligned}
$$

so that as $\left(v_{1}, v_{2}, v_{3}\right) \rightarrow\left(\widetilde{u}^{(12)}, \widetilde{u}^{(21)}, 0\right)$,

$$
\begin{gathered}
\int_{\Omega}\left[\sigma_{2}-b_{22}(x) v_{2}-b_{21}(x)\left(v_{1}-\tilde{u}^{(13)}\right)\right. \\
\left.-b_{23}(x)\left(v_{3}-\tilde{u}^{(31)}\right)\right] \psi_{2} v_{2} d x \\
\times\left(\int_{\Omega} \psi_{2} v_{2} d x\right)^{-1} \longrightarrow 0 .
\end{gathered}
$$

For the third ratio of integrals, as $\left(v_{1}, v_{2}, v_{3}\right) \rightarrow\left(\tilde{u}^{(12)}\right.$, $\left.\tilde{u}^{(21)}, 0\right)$,

$$
\begin{gathered}
\sigma_{3}-b_{33}(x) v_{3}-b_{31}(x)\left(v_{1}-\tilde{u}^{(12)}\right)-b_{32}(x)\left(v_{2}-\tilde{u}^{(21)}\right) \\
\longrightarrow \sigma_{3}>0
\end{gathered}
$$

Hence we have

$$
\liminf _{\substack{\left(v_{1}, v_{2}, v_{3}\right) \rightarrow\left(\tilde{u}_{u}^{(12)}\right) \tilde{u}^{(12), 0)} \\\left(v_{1}, v_{2}, v_{3}\right) \in X \backslash S}} \sigma^{*}\left(v_{1}, v_{2}, v_{3}\right) \geq \sigma_{3}>0 .
$$

Using the same method, we can get the conclusion about the other two cases.

$$
\begin{aligned}
& \text { As }\left(v_{1}, v_{2}, v_{3}\right) \rightarrow\left(\widetilde{u}^{(13)}, 0, \widetilde{u}^{(31)}\right), \\
& \liminf _{\substack{\left(v_{1}, v_{2}, v_{3}\right) \rightarrow\left(\tilde{u}^{(13)}, 0,\left(^{(31)}\right) \\
\left(v_{1}, v_{2}, v_{3}\right) \in X \backslash S\right.}} \sigma^{*}\left(v_{1}, v_{2}, v_{3}\right) \geq \sigma_{2}>0 .
\end{aligned}
$$

and as $\left(v_{1}, v_{2}, v_{3}\right) \rightarrow\left(0, \tilde{u}^{(23)}, \tilde{u}^{(32)}\right)$,

$$
\liminf _{\substack{\left(v_{1}, v_{2}, v_{3}\right) \rightarrow\left(0, \tilde{u}^{(23)}, \tilde{u}^{(32)}\right) \\\left(v_{1}, v_{2}, v_{3}\right) \in X \backslash S}} \sigma^{*}\left(v_{1}, v_{2}, v_{3}\right) \geq \sigma_{1}>0 .
$$

In conclusion

$$
\liminf _{\substack{\left(v_{1}, v_{2}, v_{3}\right) \rightarrow \omega(S) \\\left(v_{1}, v_{2}, v_{3}\right) \in X \backslash S}} \sigma^{*}\left(v_{1}, v_{2}, v_{3}\right)>0
$$

so that $\alpha\left(t,\left(u_{1}, u_{2}, u_{3}\right)\right)>1$ for $\left(u_{1}, u_{2}, u_{3}\right) \in \omega(S)$ and $t$ sufficiently small, and permanence follows from the abstract results of the previous section.

Now we are ready to prove an interesting result, namely, permanent existence always holds among three "very weak" competing species. The result may be known, but to our knowledge, it does not appear in the published literature.
Theorem 12. Suppose that $a_{i}(x)>\mu_{i} \lambda_{1}, b_{i i}(x)>0$ for $x \in \bar{\Omega}$, $i=1,2,3$. Then there is a constant $\delta>0$, such that if $b_{i j}<\delta$ for $i, j=1,2,3$ with $i \neq j$, the semiflow on $\left[C_{0}^{1}(\bar{\Omega})\right]^{3}$ generated by (1) under homogeneous Dirichlet boundary conditions is permanent.

Proof. For fixed $a_{i}>\mu_{i} \lambda_{1}$ and $b_{i i}(x)>0$ in $\bar{\Omega}, i=1,2,3$, when $b_{i j} \geq 0(i, j=1,2,3, i \neq j)$ are sufficiently small, conditions (11)-(13) and (17)-(19) are satisfied, and by the variational expression of principal eigenvalues (see [13]) and the boundedness of $\widetilde{u}^{(i j)}(i \neq j)$ (see Remark 8), the principal eigenvalues $\sigma_{1}, \sigma_{2}$, and $\sigma_{3}$ of (30), (31), and (32), respectively, are all positive. And then it is easy to see that condition (33) is satisfied when $b_{i j}(i \neq j)$ are even smaller, if necessary. The proof is completed by Theorem 11 .

\section{Summary and Discussion}

The important aspect of the approach of our main result, Theorem 11, is that it produces conditions that in terms of coefficients describing the strength of interactions. The criterions depend only on the behavior near the species equilibria of the two-dimensional subsystems, which is relatively easy to examine experimentally. The eigenvalues depending on the coefficients can be computed explicitly in simple cases, and there is a huge literature available on their estimation, numerical approximation, qualitative properties, and so on. Eigenvalue-based criteria for coexistence can then be used to reach biological conclusions that have been difficult to deduce through other methods.

Theorem 12 tell us that if the intercompeting coefficients are sufficiently small, the system (1) is always permanent, provided that $a_{i}>\mu_{i} \lambda_{1}$ in $\bar{\Omega}$, which is, by Lemma 5 , every single species can survive when the other two species become extinct. This sounds reasonable, and we guess that this is true for systems of any finite multiple competing species. A related topic on the systems of finite multiple competing species with constant coefficients and Neumann boundary condition can be found in [17], Theorem 9 .

The significant feature of our conditions for permanence is that they do not require any special assumptions the uniqueness of coexistence states, the existence of a global Lyapunov function, or other such properties, and so the methods used here can be used to other more general situations.

\section{Conflict of Interests}

The authors declare that there is no conflict of interests regarding the publication of this paper.

\section{Acknowledgments}

The authors thank the anonymous reviewers for their helpful comments and suggestions. This work was supported by Grant 13ZR1430100 from the Natural Science Foundation of Shanghai, China. 


\section{References}

[1] E. N. Dancer and Y. H. Du, "Positive solutions for a threespecies competition system with diffusion. I. General existence results," Nonlinear Analysis: Theory, Methods \& Applications, vol. 24, no. 3, pp. 337-357, 1995.

[2] E. N. Dancer and Y. H. Du, "Positive solutions for a threespecies competition system with diffusion, part II. The case of equal birth rates," Nonlinear Analysis: Theory, Methods \& Applications, vol. 24, no. 3, pp. 359-373, 1995.

[3] K. Kishimoto, "The diffusive Lotka-Volterra system with three species can have a stable nonconstant equilibrium solution," Journal of Mathematical Biology, vol. 16, no. 1, pp. 103-112, 1982.

[4] Y. Lou and D. Munther, "Dynamics of a three species competition model," Discrete and Continuous Dynamical Systems, vol. 32, no. 9, pp. 3099-3131, 2012.

[5] O. Vasilyeva and F. Lutscher, "Competition of three species in an advective environment," Nonlinear Analysis: Real World Applications, vol. 13, no. 4, pp. 1730-1748, 2012.

[6] R. M. May and W. J. Leonard, "Nonlinear aspects of competition between three species," SIAM Journal on Applied Mathematics, vol. 29, no. 2, pp. 243-253, 1975.

[7] P. van den Driessche and M. L. Zeeman, "Three-dimensional competitive Lotka-Volterra systems with no periodic orbits," SIAM Journal on Applied Mathematics, vol. 58, no. 1, pp. 227234, 1998.

[8] V. Hutson and R. Law, "Permanent coexistence in general models of three interacting species," Journal of Mathematical Biology, vol. 21, no. 3, pp. 285-298, 1985.

[9] D. Xiao and W. Li, "Limit cycles for the competitive three dimensional Lotka-Volterra system," Journal of Differential Equations, vol. 164, no. 1, pp. 1-15, 2000.

[10] R. S. Cantrell, C. Cosner, and V. Hutson, "Ecological models, permanence and spatial heterogeneity," Rocky Mountain Journal of Mathematics, vol. 26, no. 1, pp. 1-35, 1996.

[11] R. S. Cantrell, C. Cosner, and V. Hutson, "Permanence in some diffusive Lotka-Volterra models for three interacting species," Dynamic Systems and Applications, vol. 2, no. 4, pp. 505-530, 1993.

[12] R. S. Cantrell, C. Cosner, and V. Hutson, "Permanence in ecological systems with spatial heterogeneity," Proceedings of the Royal Society of Edinburgh A: Mathematics, vol. 123, no. 3, pp. 533-559, 1993.

[13] R. S. Cantrell and C. Cosner, Spatial Ecology via ReactionDiffusion Equations, John Wiley \& Sons, New York, NY, USA, 2003.

[14] A. W. Leung, Nonlinear Systems of Partial Differential Equations: Applications to Life and Physical Sciences, World Scientific, Singapore, 2009.

[15] V. Hutson, "A theorem on average Liapunov functions," Monatshefte für Mathematik, vol. 98, no. 4, pp. 267-275, 1984.

[16] A. W. Leung, Systems of Nonlinear Partial Differential Equations: Applications to Biology and Engineering, vol. 49 of Mathematics and Its Applications, Kluwer Academic Publishers, Dordrecht, The Netherlands, 1989.

[17] P. N. Brown, "Decay to uniform states in ecological interactions," SIAM Journal on Applied Mathematics, vol. 38, no. 1, pp. 22-37, 1980. 


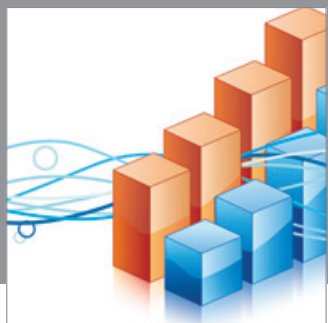

Advances in

Operations Research

mansans

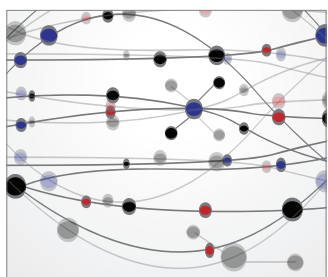

The Scientific World Journal
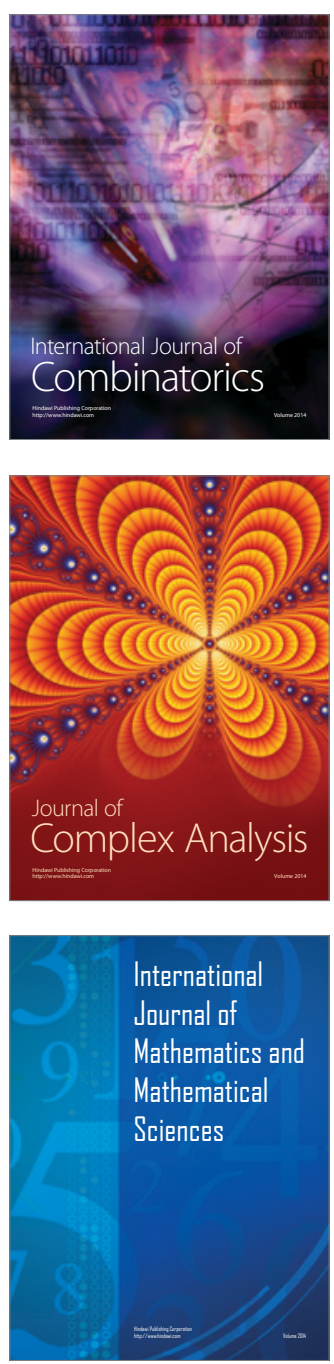
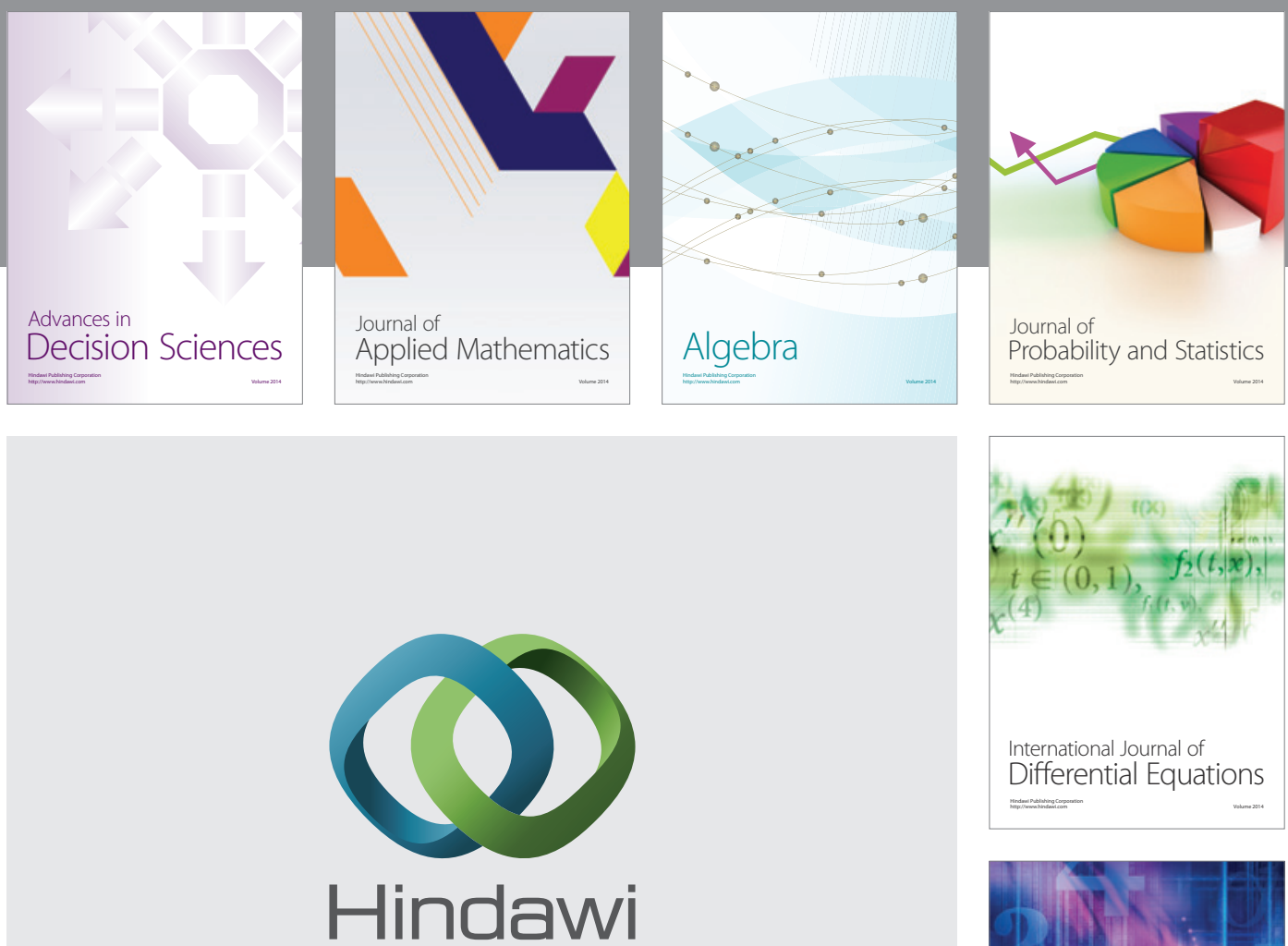

Submit your manuscripts at http://www.hindawi.com
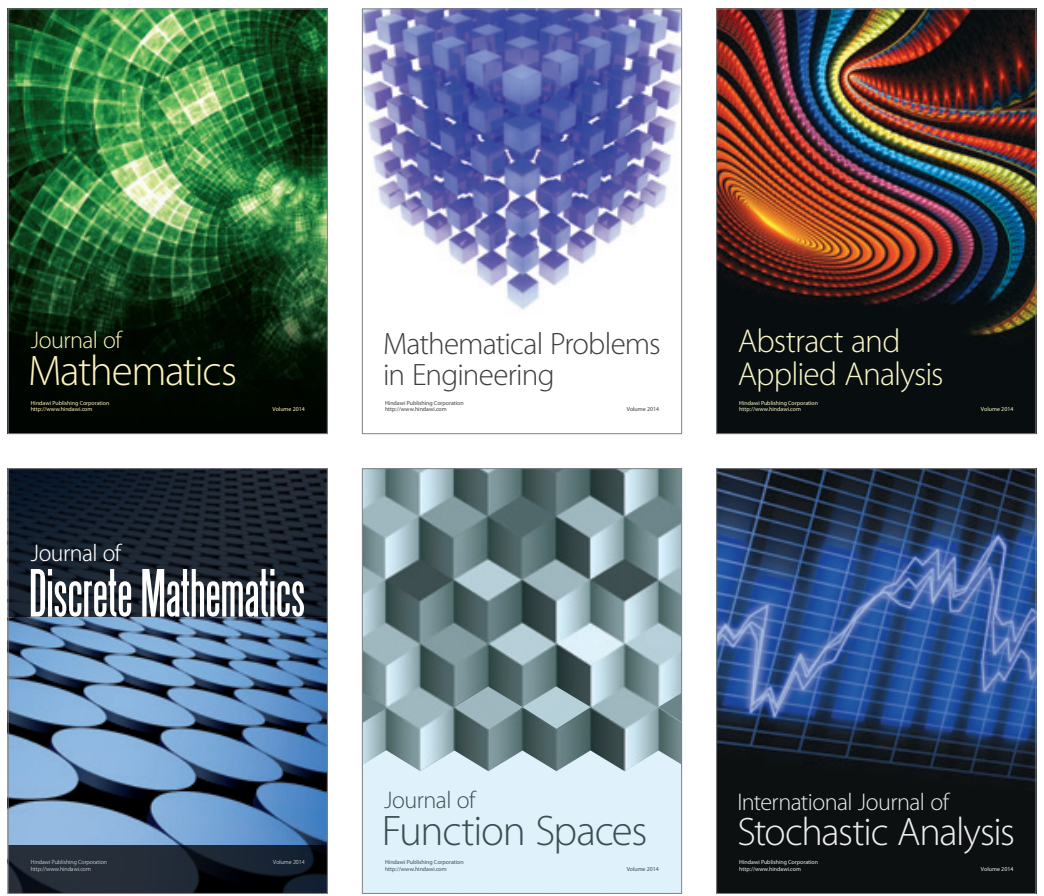

Journal of

Function Spaces

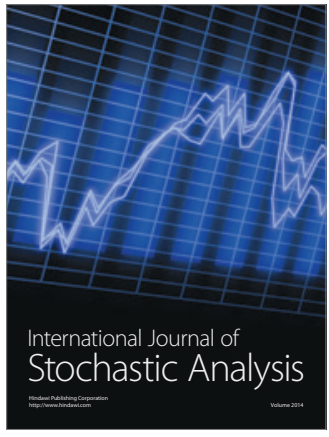

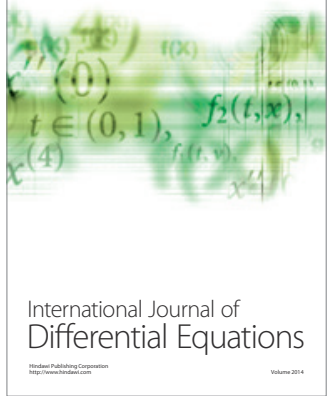
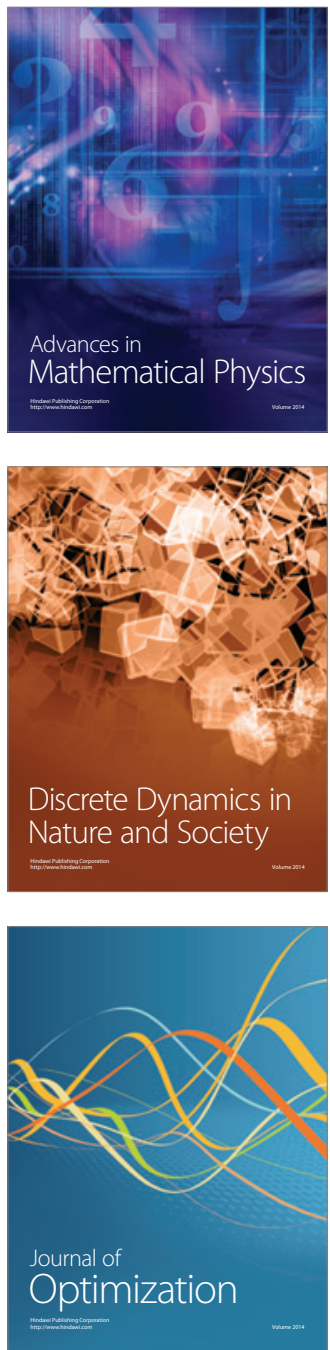